\title{
Superheat Control with a Dynamic Inverse Model
}

\author{
Andreas Varchmin ${ }^{1} \quad$ Manuel Gräber $^{2} \quad$ Wilhelm Tegethoff $^{1,2} \quad$ Jürgen Köhler $^{1}$ \\ ${ }^{1}$ Technische Universität Braunschweig, Institut für Thermodynamik \\ 2 TLK-Thermo GmbH
}

\begin{abstract}
Superheat control has influence on the coefficient of performance (COP), the stability and the compressor endurance of a vapor compression cycle. In an increasing number of applications electronic expansion valves are used. This leads to more complex control tasks. It raises the question if simulation models can be used for feedforward control to fulfill this function. For building a feedforward control structure a simulation model needs to be inverted. In this paper a continuous submodel of a refrigeration cycle, consisting of models for expansion valve and evaporator, is inverted. The resulting controller is tested in a modelin-the-loop environment and applied on an automotive refrigeration cycle. The advantage of a dynamic inverse model in contrast to a static one is pointed out. Also the results are compared to a standard PI controller.
\end{abstract}

Keywords: inverse models; superheat control; vapor compression cycles; feedforward control

\section{Introduction}

Superheat has several effects on refrigeration and heat pump cycles. The coefficient of performance (COP), the stability and the compressor endurance are dependent from a reasonable degree of superheat. Superheat control is a challenging task since the system dynamics of a refrigeration cycle are highly nonlinear and the control targets can be contrary. A higher degree of superheat leads to a smaller COP but is more secure against damage from liquid drops inside the compressor. Moreover due to the inherent characteristics of vapor compression cycles there exists an instability region at small degrees of superheat that expresses in a fluctuating temperature. This region can be described by the minimum stable superheat line (MSS) $[1,2]$. Hence, the target of control structures is to set a small degree of superheat with considering the robustness against crossing the MSS line.
In the last years electronic expansion valves (EXV) are used in an increasing number of applications, e.g. electronic vehicles. With EXVs superheat and thus stability and COP can be controlled directly in contrast to thermostatic expansion valves (TXV) or orifice valves. New control approaches can benefit from this additional degree of freedom.

In the last decade a lot of effort has been made to build models for describing the behavior of vapor compression cycles. Model libraries like TIL or Air Conditioning have been developed to simulate various systems. It seems consequential to use this knowledge not only for simulation but also for control. One approach for model based control is inverting the simulation models.

Using inverse models for feedforward control is common in control theory [3]. Inverting a model means to swap inputs and outputs and reform the model equations so that the inputs can be calculated when having knowledge of the outputs. In this way the actuating variables can be calculated depending of the desired system output. Modelica is convenient for inverting models since it is an equation-based language instead of signal-oriented. One of the main purposes of every modelica tool is to transform ODE- and DAE-systems. This is exactly the challenge of inverting models. For a convenient introduction in inverting models in Modelica see [4].

Often static models are used for feedforward control. In this paper a dynamic model is proposed for controlling the superheat. This has advantages if the desired system output is not a constant or if measurable dynamic disturbances act on the system.

\section{Structure of the Paper}

In section 2 a continuous system model of the refrigeration cycle is introduced. One part of the model, a submodel consisting of expansion valve and evaporator, is needed for feedforward control. The complete system model is used for model-in-the-loop tests and as an observer during measurement. In section 3 the 


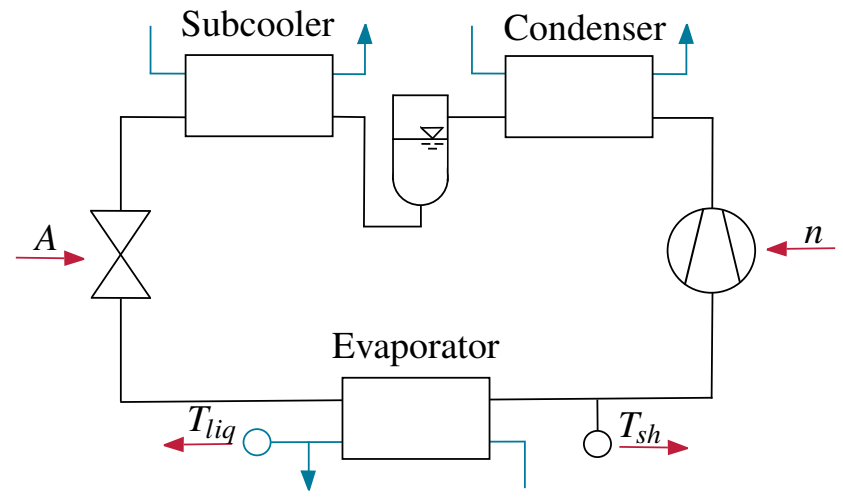

Figure 1: Modelled Refrigeration Cycle

inversion of the submodel is shown. The developed inverse model is tested in a model-in-the-loop environment in section 4 . The potential of the transient inverse model in relation to a static one is also presented. Section 5 describes the test bench setup and shows how the complete system model is used as an observer. Measurement results of the inverse feedforward control are shown in section 6 as well as closed loop control where the inverse model is coupled with a PI controller. The measurement results are compared with regular PI control results.

\section{System Model of the Refrigeration Cycle}

The modelled system is a basic refrigeration cycle consisting of a compressor, an EXV, three plate heat exchangers and a receiver (see figure 1). R134a is used as refrigerant. The compressor is a variable speed scroll compressor. On the high pressure level there are two heat exchangers: condenser and subcooler. In between lies the receiver to ensure that subcooling exists in all operating points. In the outlet of the evaporator the degree of superheat is measured. A 50/50 mixture of water and glycol flows in the secondary paths of all heat exchangers. In terms of control the valve opening $A$ and the compressor speed $n$ can be defined as inputs and the actual degree superheat $T_{s h}$ and the temperature of the cooled liquid $T_{l i q}$ as outputs.

Most of the model approaches are based on the model library TIL (see [5]). For the inversion new models with functions and interfaces were developed. All physical properties are calculated with TILMedia by use of refrigerant and liquid objects or explicit function calls (see [6]).

The dynamic compressor model is loss-based and has a suction and a discharge volume where the phys-

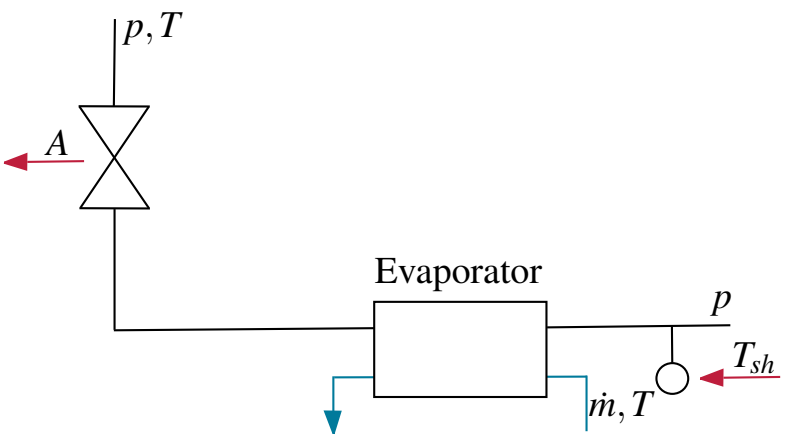

Figure 2: Inverse Model

ical properties of the refrigerant are calculated. The model parameters are fitted to the compressor in the test bench described in section 5. The EXV model originates from Bernoulli's principle and is therefore a static model. Delays that may come from the actuator of the valve are not considered even if they can not be neglected. Section 3 deals with this problem. All heat exchangers are modelled with a variable number of finite volumes. Per volume exist three cells, one for the refrigerant, one for the liquid and one for the wall dividing the fluid paths. The refrigerant cell includes transient mass, energy and momentum balances. The time derivative of pressure is constant over all refrigerant cells of a pressure level [7]. The liquid cell includes transient balances for mass and energy. Pressure drop is neglected in both liquid and refrigerant cells. The wall cell includes a transient energy balance. The receiver between the two high pressure heat exchangers is modelled as a volume with transient mass and energy balances and a varying filling level.

\section{Inverting the Submodel}

For representing the complete system dynamics for feedforward control it is desirable to invert the whole system model described in the previous section. With linear analysis of the refrigeration cycle it can be shown that an inversion of the complete model is not reasonable due to possible non-minimum phase characteristics. This would lead to an unstable system model. Hence, in this paper only a submodel is used for feedforward control. The inverted model consists of the evaporator and valve models.

The resulting inverse model is shown in figure 2 . The easiest way creating it is using the MSL Block InverseBlockConstraints that changes in- and outputs. The desired superheat $T_{s h}$ is now an input and the resulting valve opening $A$ an output. The outlet liquid temperature of the evaporator, that is equivalent to 
the cooling capacity, shall be controlled by the compressor speed and is therefore not needed for superheat control. Since the inverse model does not include the whole system dynamics boundary conditions are needed. One combination of possible boundaries is shown in figure 2. High and low pressure as well as the refrigerant inlet enthalpy of the valve are needed to simulate the model. Moreover the mass flow rate and temperature of the evaporator inlet need to be known. This information can be provided by simulation data when using the inverse model in a model-in-the-loop environment. For use as a hardware controller measurement data and/or data from an observer can be used (see section 5).

The valve opening is dependent from the opening actuator time delay. This delay can be approximated as a first-order time delay. Reforming the ODE system gets more complicated because of an additional continuous state that makes it necessary to derive more equations and to give access to second derivatives with respect to time of physical properties. For these reasons including the valve actuator delay was not possible at the time of writing this paper.

The inverse model forms an ODE with 15 continuous states. For calculating the saturation temperature dependent from pressure, that is needed for calculating the superheat, a function from TILMedia is used. Also the time derivative of this function is provided. For comparison also a (nearly) stationary inverse model is build. When eliminating the continuous states several systems of nonlinear of equations result. To prevent these hardly solvable systems the capacities of the continuous states are chosen very small so that a nearly stationary model is generated.

\section{Simulation Results}

This section starts with results regarding the sole inverse model and continues with model-in-the-loop (MiL) tests. In figure 3 the response of the inverse model to a change of the superheat setpoint is shown. The superheat follows the reference trajectory besides a small difference. This comes from a filter that smoothes the reference trajectory to make it differentiable. Otherwise the model would not be invertible (see also [4]). The actuating variable, the valve opening area, fluctuates mainly at the changes of the reference trajectory derivative at $0 \mathrm{~s}$ and $5 \mathrm{~s}$. A real-world EXV may not be able to follow the actuator trajectory. The high peaks, especially at $5 \mathrm{~s}$ and $6 \mathrm{~s}$, are mostly important to follow the gradient of the set point change.
In most applications it is not necessary to follow this gradient exactly.
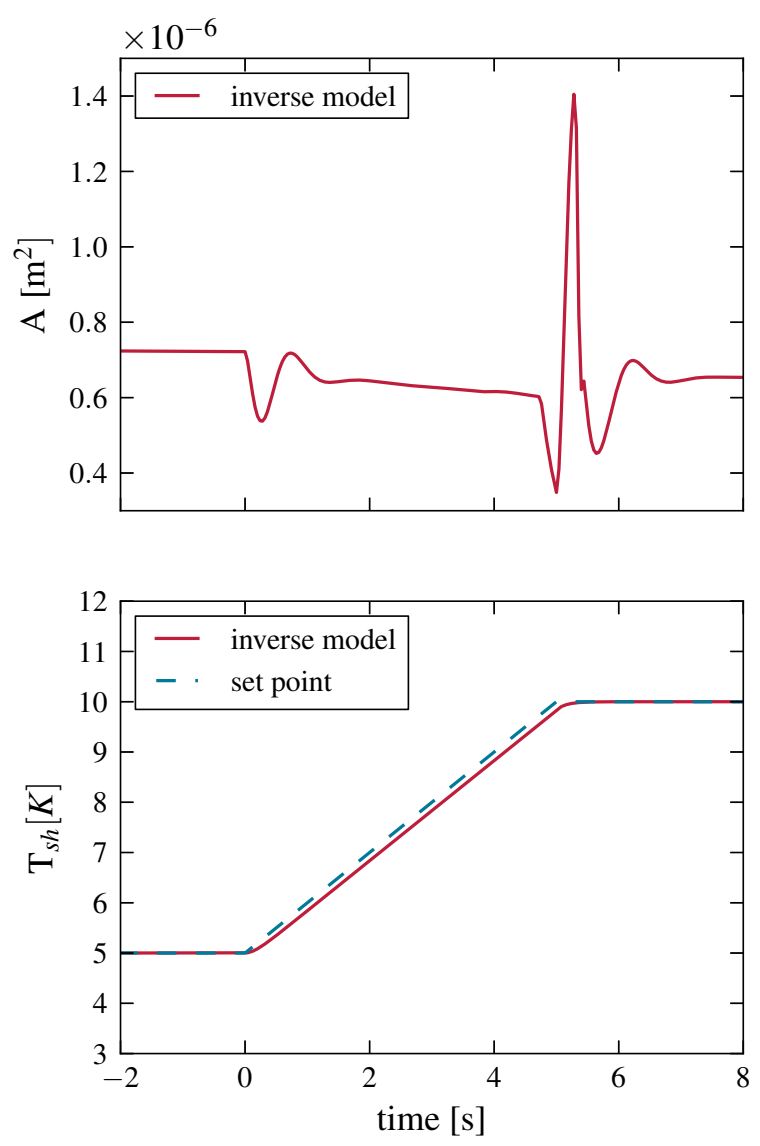

Figure 3: Behavior of Inverse Model to Setpoint Change of Superheating Degree

Figure 4 shows a set point change in a MiL environment. A transient and a static inverse model work as feedforward controllers in this example. It is obvious that the transient model can make the system model follow the reference trajectory a lot better. The static model is not able to represent the dynamics of the system and can not follow the reference trajectory. In both simulations the set point is not reached exactly. This arises from model uncertainties between inverse and system models. The valve opening fluctuates more than in the previous simulation of the sole transient model. Because of the two pressures and the valve inlet enthalpy there exists a coupling between the whole system model and the inverse model in MiL simulations. This leads to a more dynamic system. Summarizing the transient feedforward control offers a big potential for the response to setpoint changes.

Besides the response to setpoint changes the behavior towards disturbances is a main attribute for reliable and robust control. One of the advantages of feedfor- 

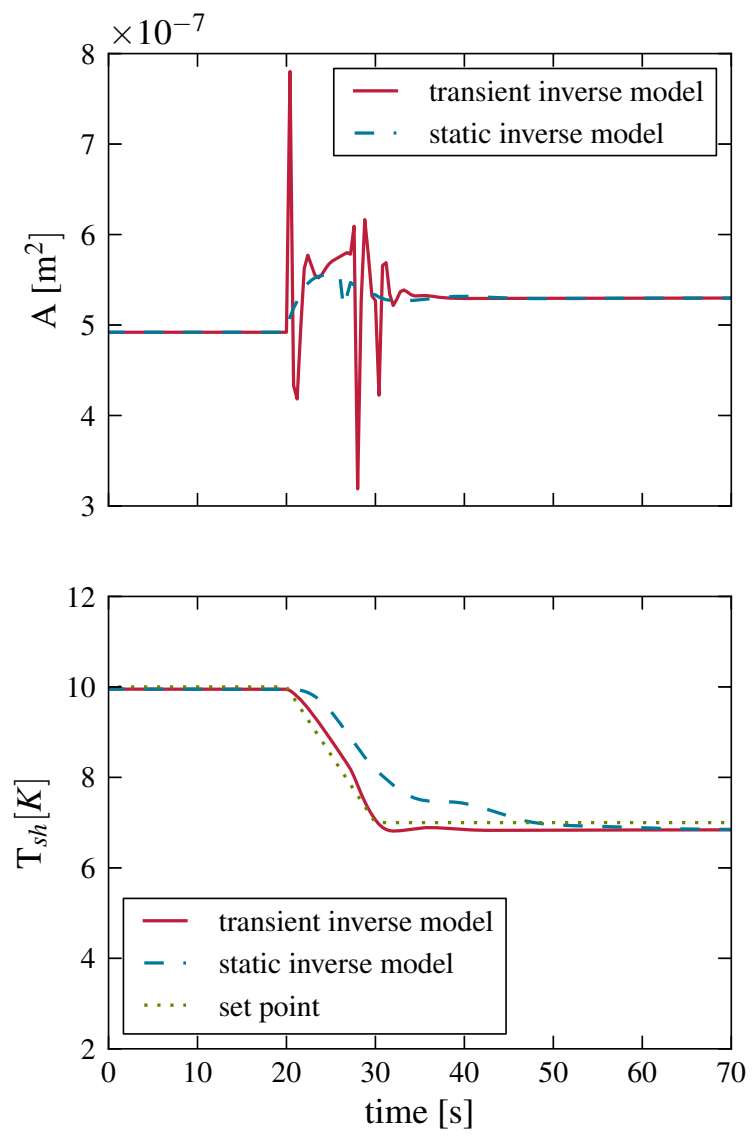

Figure 4: Comparison between Static and Transient Inverse Model Control (Model-in-the-Loop)

ward control combined with feedback control is that these behaviors can be divided. Mostly the feedforward part is mainly responsible for responses to setpoint changes so that the feedback part can be designed for reacting on disturbances and model uncertainties. If the disturbances are measurable also feedforward control can handle them and even improve the control scheme. In the described process model disturbances can be imagined as changes in input temperatures of the secondary loop cycles or as changes in the compressor speed. The compressor speed can be controlled as well and is therefore known. A change in compressor speed has influence on pressures and mass flow rate of the refrigerant cycle. This information is provided to the inverse model that can react on this disturbance. An example is shown in figure 5 where the compressor speed is raised from $2000 \mathrm{rpm}$ to $3000 \mathrm{rpm}$ at time 0. Results are shown for feedforward control including a model uncertainty and a PI controller. The PI controller reacts as recently as the measurement signal deviates from the set point. The superheat rises from $10 \mathrm{~K}$ to approximately $15 \mathrm{~K}$. The feedforward controller reacts at time 0 when the compressor speed rises. The superheat stays under $11 \mathrm{~K}$. Even if the PI controller could be optimized further for reacting on this disturbance it can never react as fast and precise as the inverse model.
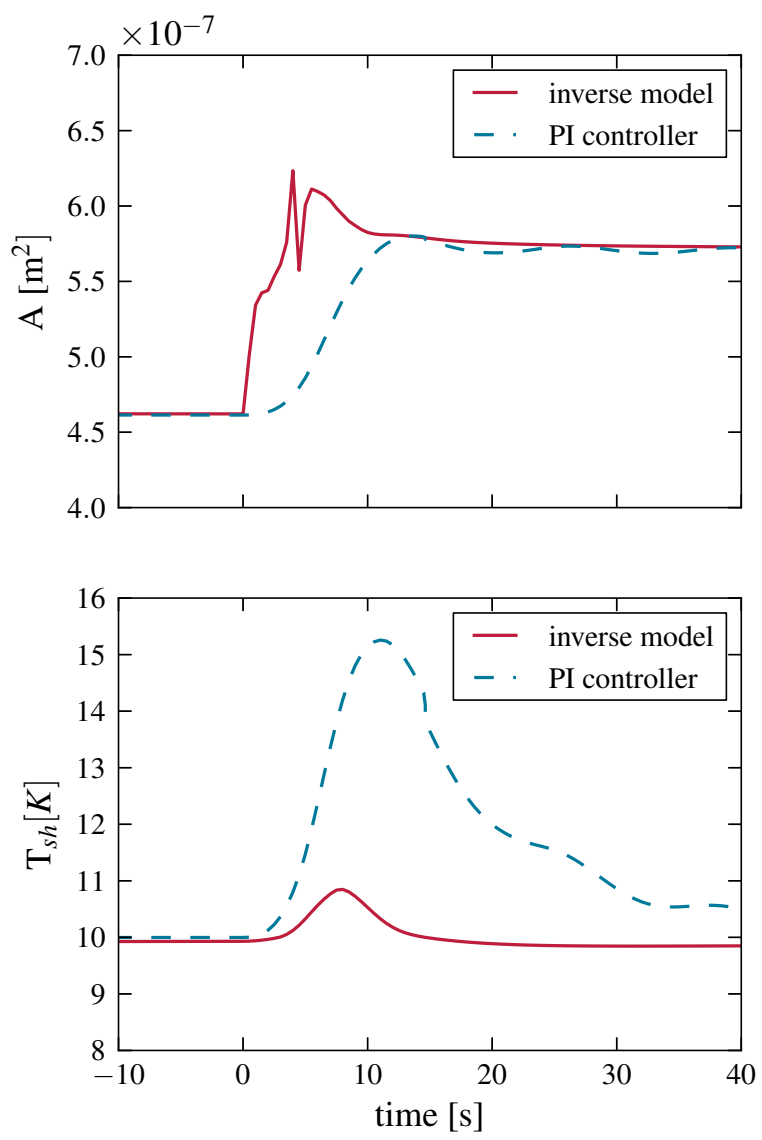

Figure 5: Behavior of the System Model towards Disturbance (Change of Compressor Speed): Comparison between Inverse Model Control and PI Controller

\section{Test Bench Setup}

The developed controller is tested on a refrigeration cycle that has the dimension of an automotive air conditioning application (see figure 6). The compressor is a direct current scroll compressor with variable speed as it is used in electric vehicles. As heat source and sink controllable secondary loop cycles filled with a glycol water mixture are connected with the plate heat exchangers. The EXV is driven by a stepper motor with constant adjusting speed. The motor needs a voltage signal for positioning the actuator. The voltage is calculated by a linear correlation with the valve opening area as input. The degree of superheated is measured with two thermocouples, one at the inlet and 
one at the outlet of the evaporator. The difference of the measured temperatures will equal the superheat if there exists two-phase refrigerant at the inlet which is the case during all measurements. The measured inlet temperature can be verificated by the measured inlet pressure that is bound to the evaporation temperature.

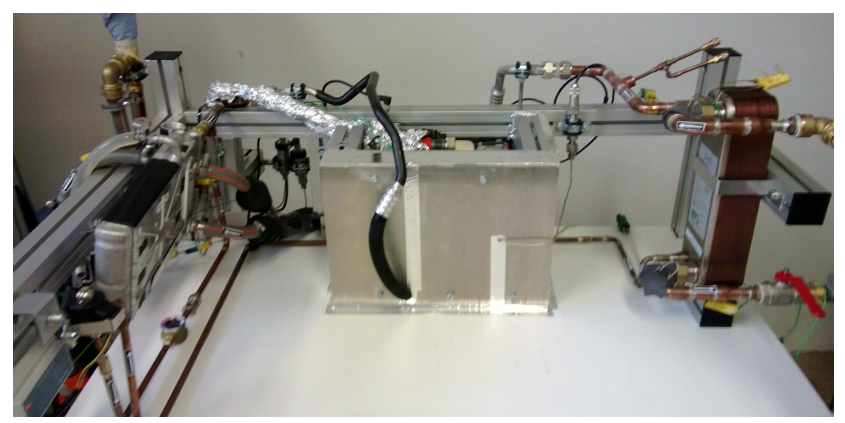

Figure 6: Test Bench with compressor, evaporator, condenser and expansion valve

A system model of the complete cycle (see section 2 ) is used as an observer. The current compressor speed and measured liquid temperatures and mass flow rates at evaporator, condenser and subcooler are made available to the observer. With this information it calculates high and low pressure and the valve inlet enthalpy that are needed by the inverse submodel.

The controller, consisting of the described inverse model for feedforward control and an optional PI controller for closing the control loop, and the observer are modelled in Dymola. The control scheme is shown in figure 7. Dymola is coupled with Labview in a cosimulation environment. Labview reads the measurement data and sends process inputs, e.g. valve opening area. One time step has a length of $0.1 \mathrm{~s}$.

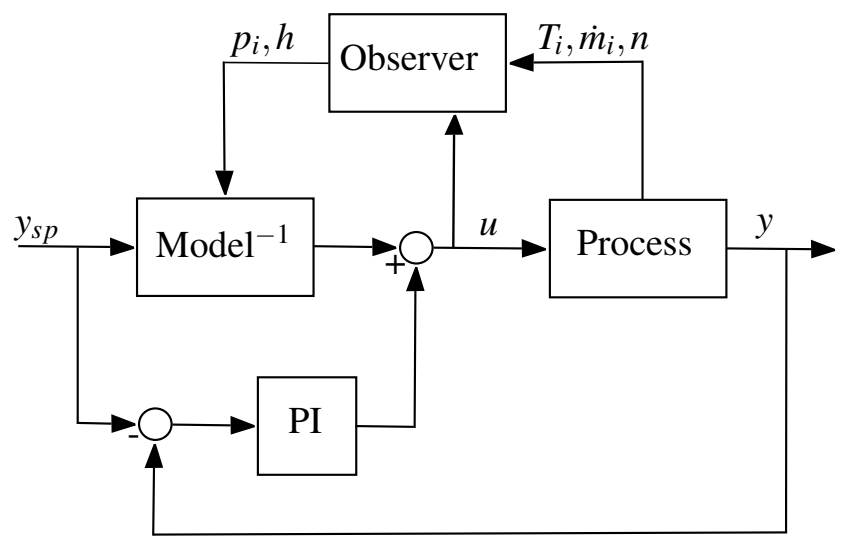

Figure 7: Inverse Model in Combination with PI Controller for Closed Loop Control

\section{Measurement Results}

In this section the developed model-based controller is applied to the test bench described previously. Results with the lone inverse model and combined with an PI controller are shown. For comparison also results generated with a lone PI controller are shown.
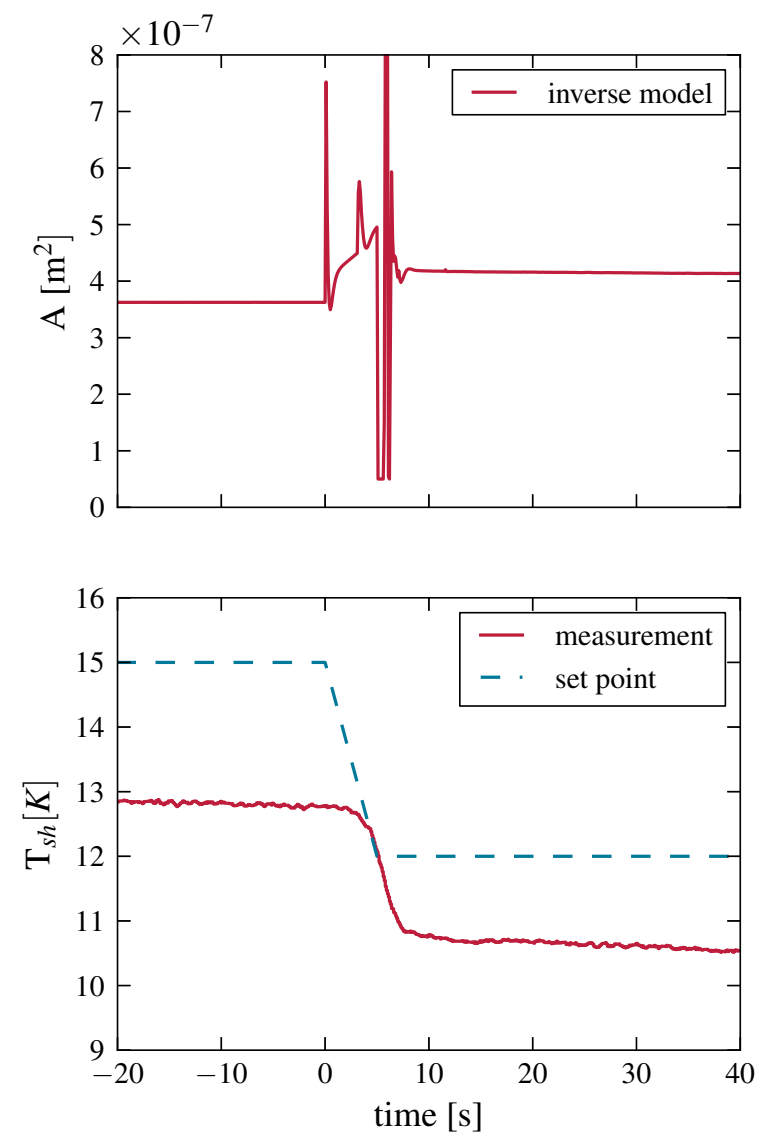

Figure 8: Comparison of model-based controller to setpoint change

In figure 8 the refrigeration cycle behavior to a set point change is shown. The setpoint follows a five second lasting ramp from $15 \mathrm{~K}$ superheat to $12 \mathrm{~K}$. At $0 \mathrm{~s}$ the degree of superheated lies at approximately $13 \mathrm{~K}$. The deviation arises from model uncertainties. In particular the uncertainties come mainly from a differing mass flow and unexact calculation of the heat transfer coefficients. Since no feedback loop is implemented in this case there is a stationary deviation. The sensor value drops nearly as fast as the setpoint and reaches its stationary value after circa six seconds. The measured superheat change is smaller than the demanded which also results from the described model uncertainties. The shown valve opening area does not picture the achieved opening area but the demand of the in- 
verse model. The high peaks arise from the harsh bend in the set point ramp and the missing actuator delay in the inverse model. These peaks are filtered before directing the signal to the valve. Omitting the peaks the valve opening area rises nearly linear, then drops to a point beneath the new stationary value und rises again to the new stationary value. Summarizing apart from an error in stationary values the inverse model feedforward controller can change the degree of superheat fast and as demanded.

In the following the feedforward controller is combined with a PI controller to compensate the mentioned model uncertainties. There exist several rules to design the parameters of a PI controller when combined with feedforward control. In this case the feedforward controller gets information from the observer, especially the actual pressures. This means that the inverse model, even if called feedforward controller in this paper, has a kind of feedback loop implemented. High and low pressure react on a changing valve opening which for its part has influence on pressures. Hence, designing the PI controller is more challenging and standard design rules can not be used without adaptation. In this paper the parameters of the PI controller for combination with the inverse model are therefore iteratively chosen and will not be optimal.

For comparison with a standard PI controller typical design rules can be used for parametrization. In this paper the from Åström and Hägglund as a ZieglerNichols replacement introduced $A M I G O$ method [8] is applied. For using this approach a measured step response is needed. The aim is to maximize the integral gain by modeling the step response with a dead time and a first-order block. Hence, three parameters have to be tuned to represent the step response. With knowledge of these parameters the PI control parameters can be obtained by simple, explicit equations.

In figure 9 a comparison between the developed model-based controller and a lone PI controller is shown. Compared to the lone feedforward control (fig. 9) the measured superheat meets the set point since the integral gain can compensate model uncertainties. The developed controller is able to drop the superheat more quickly but does undershoot the new setpoint a bit. This probably comes from the non-optimal cooperation of inverse model and PI controller. Improving this could lead to a fast and sharp response as with the lone model-based controller. The PI controller is slower but does less undershooting. It is important to mention that the PI controller is tuned for this exact operating point. Providing this quality for other operating points would mean to have an at least two-dimensional gain scheduling to compensate different compressor speeds and evaporator inlet liquid temperatures. The actuating variable in figure 9 is shown as voltage since the lone PI controller does not give an opening area value. It can be seen that the model-based controller can calculate a more transient actuator signal.
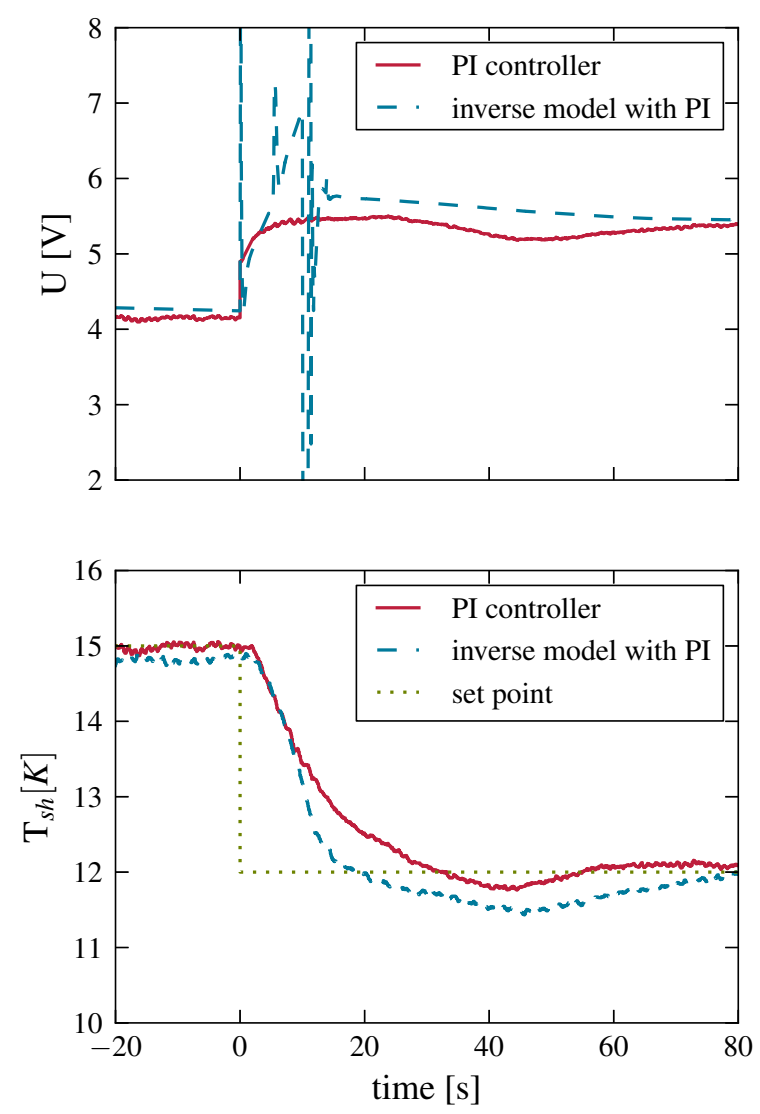

Figure 9: Comparison between reactions of modelbased controller (inluding PI controller) and PI controller to setpoint change

\section{Conclusion}

In this paper a superheat controller based on an inverse model of valve and evaporator was developed and tested in simulations and measurements. The controller can react faster on set point changes than an optimized PI controller. The biggest advantage is the compensating reaction on measurable disturbances like changing compressor speed / cooling capacity. In contrast to PI control the model-based controller works in several operating points without parameter changes or gain scheduling. Further improvements 
can be accomplished by including the valve actuator delay in the inverse model. Furthermore the interaction with a PI controller for compensating on model uncertainties and non-measurable disturbances can be enhanced.

\section{Acknowledgements}

This work has been supported by the German Ministry BMBF in the Reflex Thermo project.

\section{References}

[1] Y. Chen, S. Deng, X. Xu and M. Chan. A study on the operational stability of a refrigeration system having a variable speed compressor. International Journal of Refrigeration, 31(8):13681374, May 2008.

[2] W. Chen, Z. Chen, R. Zhu and Y. Wu. Experimental investigation of a minimum stable superheat control system of an evaporator. International Journal of Refrigeration, 25(8):11371142, December 2002.

[3] K.J. Åström and T. Hägglund. Advanced PID Control. Instrumentation, Systems and Automation Society, USA, August 2005.

[4] G. Looye, M. Thümmel, M. Kurze, M. Otter, J. Bals. Nonlinear Inverse Models for Control. In: Proceedings of the 4th International Modelica Conference, Hamburg, Germany, 2005.

[5] C. Richter. Proposal of New Object-Oriented Equation-Based Model Libraries for Thermodynamic Systems. PhD thesis, Technische Universität Braunschweig, 2008.

[6] C. Schulze. A Contribution to Numerically Efficient Modelling of Thermodynamic Systems. PhD thesis, Technische Universität Braunschweig, 2013.

[7] M. Gräber, K. Kosowski, C. Richter, W. Tegethoff. Modelling of Heat Pumps with an ObjectOriented Model Library for Thermodynamic Systems. Mathematical and Computer Modelling of Dynamical Systems, Taylor \& Francis, October 2010.

[8] T. Hägglund, K.J. Åström. Revisiting the Ziegler-Nichols rules for PI control. Asian Journal of Control, 4(4):364-380, 2002. 